Arch. Tierz., Dummerstorf 46 (2003) 3, 269-272

Aus der Thüringer Landesanstalt für Landwirtschaft Jena, Abteilungen Tierproduktion ${ }^{1}$ und Untersuchungswesen ${ }^{2}$

WERNER REICHARDT ${ }^{1}$ und BÄRBEL ECKERT ${ }^{2}$

\title{
Methodenvergleich zur Fettextraktion aus Rückenspeck bei der Bestimmung der Fettsäurenzusammensetzung und der Iodzahl
} (Kurzmitteilung)

Herrn Professor Dr. Dr. h. c. Klaus Ender zum 60. Geburtstag gewidmet

\begin{abstract}
Title of the paper: Method comparison to the fat extraction from back bacon at the determination of the fatty acid composition and the iodine value (short communication)

Trichlormethane, n-hexane as pure solvents and volume-per-volume mixtures of trichlormethane-methanol 3:1, of dichlormethane-methanol 9:1 as well as 2:1 and of n-hexane-acetone 2:1 are used to extract fat from the outer layer of back bacon of pigs by means of ultra sound. From the analysis of the iodine value according to WIJS at 15 animals arose, that under the chosen conditions the n-hexane-acetone mixture expelled the highest iodine value and the narrowest correlation to the iodine value, which was calculated from the fatty acid composition. There were significant differences at the polyunsaturated fatty acids only between n-hexane and the other extraction solvents or mixtures.
\end{abstract}

Key Words: pig, back bacon, fat extraction, fatty acid pattern, iodine value, comparison of methods

\section{Zusammenfassung}

Zur Kaltextraktion von Fett aus der äußeren Rückenspeckschicht von Schweinen mittels Ultraschall wurden Trichlormethan und n-Hexan als reine Lösungsmittel sowie volumenanteilige Mischungen Trichlormethan-Methanol 3:1, Dichlormethan-Methanol 9:1 oder 2:1 und n-Hexan-Aceton 2:1 eingesetzt. Aus der Analyse der Iodzahl nach WIJS bei 15 Tieren ergab sich, dass unter den gewählten Bedingungen die n-Hexan-Aceton-Mischung die höchste Iodzahl und die engste Korrelation zu der aus der Fettsäurenzusammensetzung errechneten Iodzahl aufwies. Bei den mehrfach ungesättigten Fettsäuren bestanden nur zwischen n-Hexan und den anderen Extraktionsmedien signifikante Unterschiede.

Schlüsselwörter: Schwein, Rückenspeck, Fettextraktion, Fettsäurenmuster, Iodzahl, Methodenvergleich

1.

Einleitung

Das Fettsäurenmuster des Rückenfetts von Schweinen sowie die Fett- oder Iodzahl (IZ) als summarischer Parameter des Gesamtanteils der $\mathrm{C}=\mathrm{C}$-Doppelbindungen an Fetten und Ölen sind seit Jahren Gegenstand zahlreicher Untersuchungen. Einerseits beeinflussen die Fettsäuren als Bestandteil menschlicher Nahrung die Gesundheit, andererseits bestimmen sie Haltbarkeit, Konsistenz und Geschmack von Produkten wie Dauerwurst. Im Rahmen einer mehrjährigen Erhebung zum Fettsäurenmuster von Rückenfett bei Thüringer Schweinen sollte auch der Einfluss des Extraktionsmediums auf die einzelnen Kennwerte geprüft und eine Alternative zum Einsatz von stark toxischen (Trichlormethan $>$ Dichlormethan) und umweltschädigenden Halogenkohlenwasserstoffen gefunden werden. Daher wurde anstelle des oft genutzten Extraktionsmediums 
Trichlormethan-Methanol 2:1 (v/v; FOLCH u.a., 1957) die in Schweizer Schlachthöfen bei der Ermittlung der Fettzahl von Rückenfett verwendete Mischung von Dichlormethan-Methanol 2:1 (v/v; GLÄSER, 2000) in den Vergleich einbezogen. Nach ARNETH (1998) unterscheidet sich die Polarität $\varepsilon^{\circ}$ und damit die Fähigkeit zur Lipidextraktion beider Halogenkohlenwasserstoffe kaum (an Aluminiumoxid: $\varepsilon^{\circ}-\mathrm{CHCl}_{3}$ : 0,$\left.38 ; \varepsilon^{\mathrm{o}}-\mathrm{CH}_{2} \mathrm{Cl}_{2}: 0,42 ; \varepsilon^{\mathrm{o}}-\mathrm{CH}_{3} \mathrm{OH}: 0,95\right)$. Die geringe Differenz wird außerdem durch den Zusatz des polareren Lösungsmittels Methanol nahezu ausgeglichen. Entsprechend fanden CHEN u.a. (1981) bei einem Vergleich von Trichlormethan-Methanol 2:1 (v/v) und Dichlormethan-Methanol 2:1 (v/v) keine signifikanten Unterschiede hinsichtlich der aus verschiedenen Nahrungsmitteln (u.a. Fleisch, Wurst, Schinken) extrahierten Fettmenge.

\section{2.} Material und Methoden

Die Untersuchungen erfolgten an 15 Proben aus der äußeren Rückenspeckschicht, die über dem 13./14. Brustwirbel der rechten Schlachtkörperhälfte bei Schweinen aus der Thüringer Leistungsprüfanstalt entnommen wurden. Die methodische Ausführung der Extraktion sowie der gaschromatographischen Analyse (Vierfachbestimmung) wurde bereits beschrieben (REICHARDT u.a., 2003). Die Auswahl der Lösungsmittel bzw. ihrer Mischungen (Volumen/Volumen; v/v) orientierte sich an Literaturangaben:

- Trichlormethan: HALVARSSON und ALSTIN (1982)

- Trichlormethan-Methanol - 3:1 (v/v):

- Dichlormethan-Methanol - 9:1 (v/v):

ENDER und REICHARDT (1980)

- Dichlormethan-Methanol - 2:1 (v/v):

MAXWELL u.a. (1980)

CHEN u.a. (1981)

- n-Hexan:

- n-Hexan-Aceton - 2:1 (v/v):

International Standard ISO 1444 (1973)

HARA und RADIN (1978)

(Trichlormethan und Dichlormethan gelten als potentiell kanzerogen wirkend, Methanol als giftig und n-Hexan als neurotoxisch: QUELLMALZ, 2000; HEYKE und MATZKE, 1994)

Die Bestimmung der Iodzahl (IZ) erfolgte nach der Methode von WIJS (IUPAC 2.205, 1992) in zweifacher Ausführung. Der hierfür erforderliche Zeitaufwand begrenzte den Stichprobenumfang des Vergleichs von 6 Extraktionsmedien auf 15 Tiere. Als Kontrollstandards dienten bei der Erfassung der IZ nach WIJS Oliven-, Sonnenblumenund Leinöl, die nach MATISSEK u.a. (1992) Iodzahlen von 84, 132 und 186 aufweisen. Die IZ ist zusätzlich nach KOLAROVIC u.a. (1984) aus der Fettsäurenzusammensetzung berechnet worden. Zur statistischen Auswertung der Daten wurden multiple tTests mit versuchsbezogener Irrtumswahrscheinlichkeit von $\alpha=0,05$ nach Scheffé und nach Bonferroni und der vergleichsbezogene LSD-Test mit $\alpha=0,05$ herangezogen.

Die Ergebnisse des Extraktionsvergleichs sind in den Tabellen 1-2 zusammengefasst. Da die mehrfach ungesättigten Fettsäuren bei der Qualitätsbeurteilung von Rückenfett eine entscheidende Rolle einnehmen, müssen zunächst der Gehalt an C18:2, C18:3 oder die Summe aller mehrfach ungesättigten Fettsäuren (PUFA) zur Bewertung der Ergebnisse herangezogen werden. Signifikante Unterschiede im Fettsäurenmuster der Extrakte bestanden bei C18:2, C18:3, PUFA, der Summe aller ungesättigten Fettsäuren (UFA), der Iodzahl nach WIJS, der gaschromatographisch ermittelten IZ, der Differenz zwischen beiden IZ aber auch bei C17:0, C17:1, C18:0, C20:0, C22:0 und der 
Summe aller gesättigten Fettsäuren (SFA). Bei n-Hexan fanden sich die niedrigsten Gehaltswerte für C18:2 und C18:3, so dass es für das Untersuchungsziel als Extraktionsmedium ausschied. Alle anderen Extraktionsvarianten waren bezüglich der mehrfach ungesättigten Fettsäuren gleichwertig. Wird die nach der Methode von WIJS ermittelte IZ in die Bewertung einbezogen, ergibt sich bei der Extraktion mit n-HexanAceton 2:1 (v/v) im Mittel nicht nur die höchste IZ, sondern auch die engste Beziehung zur der aus der Fettsäurenzusammensetzung errechneten IZ (Tab. 2). BÄRLOCHER u.a. (2001) schätzten bei 155 Rückenfettproben, die mit Dichlormethan-Methanol - 2:1 (v/v) extrahiert wurden, zwischen chemisch und gaschromatographisch bestimmter Iodzahl (Fettzahl) eine Korrelation von $\mathrm{r}=0,95$.

Tabelle 1

Mittelwerte der Fettsäurenzusammensetzung von Rückenfett (Masse-\%) bei der Extraktion mit verschiedenen Lösungsmitteln (Means of fatty acid composition of backfat (mass-\%) after extraction using different solvents)

\begin{tabular}{l|c|c|c|c|c|c}
\hline Lösungsmittel / -mischung (v/v) & C14:0 & C16:0 & C17:0 & C18:0 & C20:0 & C22:0 \\
\hline Trichlormethan & $1,81^{\mathrm{a}}$ & $29,69^{\mathrm{a}}$ & $2,24^{\mathrm{b}}$ & $15,89^{\mathrm{a}}$ & $0,21^{\mathrm{a}}$ & $0,05^{\mathrm{b}}$ \\
Trichlormethan-Methanol - 3:1 & $1,80^{\mathrm{a}}$ & $29,61^{\mathrm{a}}$ & $2,22^{\mathrm{b}}$ & $15,97^{\mathrm{a}}$ & $0,22^{\mathrm{a}}$ & $0,06^{\mathrm{b}}$ \\
Dichlormethan-Methanol - 9:1 & $1,82^{\mathrm{a}}$ & $29,63^{\mathrm{a}}$ & $2,17^{\mathrm{b}}$ & $15,74^{\mathrm{a}}$ & $0,22^{\mathrm{a}}$ & $0,05^{\mathrm{b}}$ \\
Dichlormethan-Methanol - 2:1 & $1,88^{\mathrm{a}}$ & $30,34^{\mathrm{a}}$ & $1,13^{\mathrm{a}}$ & $16,44^{\mathrm{b}}$ & $0,21^{\mathrm{a}}$ & $0,03^{\mathrm{a}}$ \\
Hexan & $1,78^{\mathrm{a}}$ & $30,23^{\mathrm{a}}$ & $2,19^{\mathrm{b}}$ & $17,06^{\mathrm{b}}$ & $0,26^{\mathrm{b}}$ & $0,10^{\mathrm{c}}$ \\
Hexan-Aceton - 2:1 & $1,76^{\mathrm{a}}$ & $29,54^{\mathrm{a}}$ & $2,15^{\mathrm{b}}$ & $15,98^{\mathrm{a}}$ & $0,22^{\mathrm{a}}$ & $0,05^{\mathrm{b}}$ \\
\hline Lösungsmittel /-mischung (v/v) & $\mathrm{SFA}$ & $\mathrm{C} 16: 1$ & $\mathrm{C} 17: 1$ & $\mathrm{C} 18: 1$ & $\mathrm{C} 18: 2$ & $\mathrm{C} 18: 3$ \\
\hline Trichlormethan & $53,71^{\mathrm{a}}$ & $3,93^{\mathrm{a}}$ & $0,54^{\mathrm{b}}$ & $34,71^{\mathrm{a}}$ & $6,22^{\mathrm{b}}$ & $0,88^{\mathrm{b}}$ \\
Trichlormethan-Methanol - 3:1 & $52,86^{\mathrm{a}}$ & $4,01^{\mathrm{a}}$ & $0,52^{\mathrm{b}}$ & $35,22^{\mathrm{a}}$ & $6,50^{\mathrm{b}}$ & $0,89^{\mathrm{b}}$ \\
Dichlormethan-Methanol - 9:1 & $53,36^{\mathrm{a}}$ & $4,02^{\mathrm{a}}$ & $0,53^{\mathrm{b}}$ & $35,04^{\mathrm{a}}$ & $6,17^{\mathrm{b}}$ & $0,86^{\mathrm{b}}$ \\
Dichlormethan-Methanol - 2:1 & $53,46^{\mathrm{a}}$ & $3,95^{\mathrm{a}}$ & $0,42^{\mathrm{a}}$ & $34,91^{\mathrm{a}}$ & $6,31^{\mathrm{b}}$ & $0,93^{\mathrm{b}}$ \\
Hexan & $54,84^{\mathrm{b}}$ & $3,87^{\mathrm{a}}$ & $0,53^{\mathrm{b}}$ & $34,70^{\mathrm{a}}$ & $5,42^{\mathrm{a}}$ & $0,64^{\mathrm{a}}$ \\
Hexan-Aceton - 2:1 & $53,40^{\mathrm{a}}$ & $3,89^{\mathrm{a}}$ & $0,53^{\mathrm{b}}$ & $35,05^{\mathrm{a}}$ & $6,27^{\mathrm{b}}$ & $0,86^{\mathrm{b}}$ \\
\hline
\end{tabular}

Werte mit dem gleichen Hochbuchstaben unterscheiden sich nicht signifikant $(\alpha=0,05)$

\section{Tabelle 2}

Mittelwerte von MUFA, PUFA und UFA (Masse-\%), der Iodzahlen nach WIJS und der gaschromatographisch ermittelten Iodzahl sowie die Koeffizienten der Korrelation beider Iodzahlen bei der Extraktion mit verschiedenen Lösungsmitteln (Means of MUFA, PUFA and UFA (mass-\%), of the iodine value after the method of WIJS, the iodine value determined by gas chromatography and the coefficients of correlation of both iodine values after extraction using different solvents)

\begin{tabular}{l|c|c|c|c|c|c|c}
\hline Lösungsmittel / -mischung (v/v) & MUFA & PUFA & UFA & IZ WIJS & IZGC & $\Delta$ IZ & r \\
\hline Trichlormethan & $39,2^{\mathrm{a}}$ & $7,1^{\mathrm{b}}$ & $46,3^{\mathrm{a}}$ & $59,9^{\mathrm{a}}$ & $50,5^{\mathrm{b}}$ & $9,4^{\mathrm{b}}$ & $0,55^{*}$ \\
Trichlormethan-Methanol-3:1 & $39,8^{\mathrm{a}}$ & $7,4^{\mathrm{b}}$ & $47,2^{\mathrm{b}}$ & $58,6^{\mathrm{a}}$ & $51,4^{\mathrm{b}}$ & $7,2^{\mathrm{a}}$ & $0,66^{* *}$ \\
Dichlormethan-Methanol - 9:1 & $39,6^{\mathrm{a}}$ & $7,0^{\mathrm{b}}$ & $46,7^{\mathrm{b}}$ & $60,1^{\mathrm{a}}$ & $50,7^{\mathrm{b}}$ & $9,4^{\mathrm{b}}$ & $0,73^{* *}$ \\
Dichlormethan-Methanol - 2:1 & $39,3^{\mathrm{a}}$ & $7,2^{\mathrm{b}}$ & $46,5^{\mathrm{b}}$ & $58,5^{\mathrm{a}}$ & $51,3^{\mathrm{b}}$ & $7,2^{\mathrm{a}}$ & $0,69^{* *}$ \\
Hexan & $39,1^{\mathrm{a}}$ & $6,1^{\mathrm{a}}$ & $45,2^{\mathrm{a}}$ & $59,7^{\mathrm{a}}$ & $48,2^{\mathrm{a}}$ & $11,5^{\mathrm{c}}$ & $0,68^{* *}$ \\
Hexan-Aceton - 2:1 & $39,5^{\mathrm{a}}$ & $7,1^{\mathrm{b}}$ & $46,6^{\mathrm{b}}$ & $61,0^{\mathrm{b}}$ & $50,8^{\mathrm{b}}$ & $10,2^{\mathrm{c}}$ & $0,84^{* *}$ \\
\hline
\end{tabular}

MUFA = Summe aller einfach ungesättigten Fettsäuren; IZ WIJS= Iodzahl nach WIJS; IZGC = gas-chromatographisch ermittelte Iodzahl; $\Delta$ IZ = IZ WIJS - IZGC; $r$ = PEARSONscher Korrelationskoeffizient; Werte mit dem gleichen Hochbuchstaben unterscheiden sich nicht signifikant $(\alpha=0,05)$;

$*=\alpha=0,05 ; * *=\alpha=0,01$

4.

Schlussfolgerungen

Aufgrund der Vergleichbarkeit der erzielten Fettsäurenkennwerte, der Höhe der Iodzahl nach WIJS und ihrer guten Korrelation zu der aus der Fettsäurenzusammensetzung errechenbaren Iodzahl kann wegen der im Vergleich zu halogenierten Kohlenwasserstoffen und Methanol geringeren gesundheitsschädlichen Wirkung von n-Hexan und Aceton auch eine 2:1 (v/v)-Mischung dieser beiden Lösungsmittel zur Extraktion 
von Fett aus dem Rückenspeck von Schweinen herangezogen werden, wenn die Iodzahl und / oder die Fettsäurenzusammensetzung von Rückenfett zu bestimmen ist.

\section{Literatur}

ARNETH, W.:

Über die Bestimmung des intramuskulären Fettes. Fleischwirtsch. 78 (1998) 3, 218-220

BÄRLOCHER, H.U.; BADERTSCHER, R.; WENK, C.; SCHEEDER, M.R.L.:

Übereinstimmung von Titrationsmethode und gaschromatographischer Fettsäurenbestimmung bei der Bewertung der Fettqualität von Mastschweinen. Schriftenreihe Institut für Nutztierwissenschaften, ETH Zürich, Band 21 (2001), 168-169

CHEN, I.S.; SHEN, C.-S.J.; SHEPPARD, A.J.:

Comparison of Methylene Chloride and Chloroform for the Extraction of Fats from Food Products. J. Am. Oil Chem. Soc. 58 (1981) 5, 599-601

ENDER, K.; REICHARDT, W.:

Zur Bestimmung des Gehaltes von intramuskulärem Fett in Rindfleisch mit Hilfe der Chloroform/MethanolExtraktion. Nahrung 24 (1980) 6, 493-498

FOLCH, J.; LEES, M.; SLOANE STANLEY, G.H.:

A simple method for the isolation and purification of total lipids from animal tissues. J. Biol. Chem. 226 (1957) 497-509

GLÄSER, K.R.:

Untersuchungen zur Eignung der Fettzahl als Kriterium für die Einschätzung der Fettqualität von Mastschweinen unter dem Einfluss der Fütterung und im Hinblick auf die Qualität von Verarbeitungsprodukten. ETH Zürich, Diss., 2000

HALVARSSON, H.; ALSTIN, F.:

SOXTEC HT - ein neues, vielseitig einsetzbares Extraktions-System. Focus 5 (1982) 1, 2-4

HARA, A.; RADIN, N.S.:

Lipid Extraction of Tissues with a Low Toxicity Solvent. Analyt. Biochem. 90 (1978), 420-426

HEYKE, H.-E.; MATZKE, G.:

Hexanfreies Pentan. Nachr. Chem. Tech. Lab. 42 (1994) 1, 60

INTERNATIONAL STANDARD ISO 1444:

Meat and meat products - Determination of free fat content. 1973, 1. Ed., 1-2

IUPAC 2.205:

Determination of the iodine value. In: Standard Methods for the Analysis of oils, fats and derivatives. 1992

KOLAROVIC, L.; TRAITLER, H.; DUCRET, P.:

On-line gaschromatographic evaluation of iodine values in edible fats and oils. J. Chromatogr. 314 (1984), 233-241

MATISSEK, R.; SCHNEPEL, F.-M.; STEINER, G.:

Lebensmittelanalytik. Grundzüge, Methoden, Anwendungen. 2. Auflage, Springer-Verlag Berlin, 1992, 50

MAXWELL, R.J.; MARMER, W.N.; ZUBILLAGA, M.P.; DALICKAS, G.A.:

Determination of Total Fat in Meat and Meat Products by a Rapid, Dry Column Method. J. Assoc. Off. Anal. Chem. 63 (1980) 3, 600-603

QUELLMALZ, E.:

Hauptstoffliste: zusammengeführte Informationen zu gefährlichen Stoffen und Zubereitungen aus den wichtigsten Vorschriften. WEKA Fachverlag, Augsburg, 2000

REICHARDT, W.; GERNAND, E.; MÜLLER, S.; HARTUNG, H.; ECKERT, B.; BRAUN, B.:

Erhebungen zur Fettsäurenzusammensetzung von Rückenfett bei Thüringer Schweinen sowie zum Fett von Thüringer Knackwürsten aus dem Einzelhandel. Arch. Tierz., Dummerstorf 46 (2003) 3, 257-267

Eingegangen: 01.10.2002

Akzeptiert: 06.05.2003

Anschrift der Verfasser

Dr. habil. WERNER REICHARDT, BÄRBEL ECKERT

Thüringer Landesanstalt für Landwirtschaft

Am Rennsteig 3

D-99819 Oberellen - OT Clausberg 